bilions and the pain was intense, an attack usually lasting for more than a week. The most severe attack was in April, and at that time she was confined to bed for a fortnight, and was extremely ill; but, owing to her condition-she was then in the seventh month of her pregnancy-operation was declined. The abdomen was noted to be unusually large. On the morning of June 18th she had what seemed to be the beginning of another attack, and an opiate was given to relieve the pain. This sub-sided, but next morning her doctor was summoned early and found labour apparently progressing normally, though the patient was in rather a collapsed condition. After the birth of patient was in rather a collapsed condition. After the birth of for. On my arrival the patient was moribund. On examination by the vagina I discovered a second child lying high up in the uterus, which was quite lax. The child was turned and delivered without any difficulty; but the uterus did not contract, and was found to be firmly fixed to the liver. No manipulation could free it. There was no question of its rupture, as the wall could be felt distinctly, and the hand passed

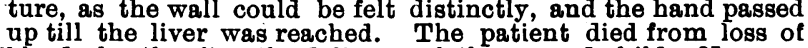
blood shortly after the delivery of the second child. No postmortem examination was allowed.

The probable course of events in this case was gallstone colic with inflammation, and in April a fistula had been formed into the colon or duodenum, and relief had been obtained at that time; but so much inflammatory adhesion had occurred that the uterus must have then become fixed to the colon or liver. At any rate, after this attack she was comparatively free from pain till her confinement in June, when the uterine contractions again caused a semblance of biliary colic.

The second case was a farm servant, J. C., aged 43, who con sulted me in April, 1902, about an attack of indigestion with extreme pain. The pain always followed ingestion of food, reaching its greatest intensity about three hours after eating, and being relieved by complete emptying of the stomach by vomiting. I advised operation, but he would not at the time consent, and he was treated by dieting and bismuth carbonate, under which he improved considerably and was able to resume work. In June of the same year the pain recurred, and there was severe haematemesis on the 12 th of that month, which so alarmed him that he consented to operation. At the operation the stomach, from the middle of the lesser curvature to the pylorus, was found to be firmly adherent to the under surface pylorus, was found to be firmly adherent to the under surface
of the liver. When these adhesions were freed a large opening was found in the anterior surface of the stomach near the pylorus, and a large eaten-out cavity in the liver from which there was persistent bleeding. This cavity measured 2 in. by 1 in., and was about $1_{\frac{1}{2}}$ in. deep. It was packed with iodoform gauze. The opening in the stomach was closed, and a drain left in the upper angle of the abdominal wound. The patient died next day. The condition was one of gastric ulcer perforating after adhesion to the liver.

The third case was seen in consultation with Dr. Macdonald of Dunkeld in November, 1908. Miss J., aged 43, had suffered from indigestion when in Ireland twelve years previously. In - Oetober she began to vomit a dark-coloured fluid. All food by the mouth was stopped, but the vomiting still persisted, amounting to several pints daily. No drug had any effect, and she got very weak and emaciated. Malignant obstruction about the pylorus was diagnosed, but very little beyond a marked resistance to palpation could be made out. The puzzling thing was the amount of fluid which must be returning through an obstructed pylorus, and which seemed to be pure bile. An alternative diagnosis was gall-stone obstruction by ulceration and blocking of the duodenum, and it was only at the operation that the diagnosis was cleared up. The gall bladder was found adherent to the front of the stomach 1 in. bladder was found adherent to the front of the stomach $1_{\text {in }}$ in.
above the pylorus, and the pylorus felt hard and fixed. The patient's condition was so poor that a posterior gastro-jejunostomy was performed. She rapidly recovered, and was enjoying light diet and sitting up in a fortnight. She then had another attack of vomiting, with marked nervousness and sleeplessness. This came on always at night; and, as nothing was found in her condition to account for it, she was allowed at her own request to go home, wh ere she again rapidly recovered, and is now quite well, and able to attend to her household duties.

In this case the ulcer may have been gastric, and have penetrated after adhesions into the gall bladder, or it may have been biliary from gall stones, and ulcerated into the stomach. In either case the condition set up pyloric inflammation and thickening with obstruction, which was relieved by the gastro.jejunostomy. It is quite likely that the inflammatory condition at the pylorus may have subsided later and the pylorus again become patent ; but, in any case, the proper treatment at the time seemed to me to be a gastro.jejunostomy. There was not much matting about the bile passages. The gall bladder adhered only at one point where the fistula existed, and nothing was discovered elsewhere to lead one to think that this case was originally one of gall stones rather than of gastric vlcer.

\section{a fatal case of acute volvulus of} THE ILEUM.

By S. E. DENYER, C.M.G., M.D., M.A.Cantab., F.R.C.S.ENG., HOLL.

I AM indebted to Dr. W. A. Bryant for giving me the opportunity of seeing and recording this case, which is of interest from a diagnostic as well as a forensic point of view.

The patient, a man aged 28 , a fitter, went to bed on the night of April 29th apparently in good health. He had done an ordinary day's work, and had not complained of any ill-health. At 3 a.m., while in bed, he was suddenly seized with acute abdominal pain and vomiting. This continued until 8 a.m., when Dr. Bryant was sent for. He saw the patient at 8.7 a.m., and found him in a state of collapse, pulseless, and with no audible heart sounds; respirations were five or six a minute, and spasmodic in character; the pupils were dilated. He gave a hypodermic injection of strychnine and digitalin, after which the pupils contracted, but there was no other reaction. The patient died at 8.15 a.m.

Dr. Bryant was told that the patient had had attacks of pain in the chest and back for some time, and his wife had recommended him to go to a doctor, but he had refused. Otherwise he had been healthy.

The body was that of a well-nourished man, looking about the stated age. There was no sign of any external injury. Rigor mortis was well marked. The muscles were found to be healthy, normal in colour, and well developed.

Heart.-Muscle and valves healthy, no sign of disease in the coronary arteries. A small quantity of clear fluid in the pericardial sac.

Lungs. - Some oedema at bases, and on the left side some old pleurisy, otherwise healthy.

Thoracic aorta healthy; no atheroma.

The brain and meninges, as well as the arteries of the brain, were healthy.

Abdomen distended; on opening the abdominal cavity a large coil of intestine was found presenting. This coil was placed vertically, and was about 20 in. in length. It was very much distended, being about the diameter of an ordinary man's coatsleeve, and was of a deep purple colour, almost black. At first, on account of its size and position, it was thought to be the transverse colon much dilated, but closer investigation showed that it was the ileum in an abnormal position. There was no peritonitis, nor any sign of perforation or gangrene. The stomach was collapsed and empty, excepting for an ounce or two of fluid contents, consisting of watery mucus with no unusual smell or appearance. The oesophagus and duodenum as well as the stomach had no sign of inflammation, corrosion, ulceration, or perforation; the mucous membrane was pale,
but otherwise normal. There were no adhesions in the neighbourhood of these organs.

As the jejunum passed into the ileum it was found to become congested and distended, becoming more so at its lower part. The ileum was distended throughout its whole extent, but mostly in its lower two feet, which presented the appearance described above. The peritoneum of the posterior abdominal wall on the right side, as it left the root of the mesentery, had 8 wall on the right side, as it left the root of the mesentery, had a dragged appearance, and the mesentery in the neighbourhood of the caecum was twisted on itself. On opening the ileum it was found to contain a large quantity of fluid faeces, and its walls were black with congested blood. One peculiarity noted was that this area of intense congestion suddenly ceased at the ileocaecal valye; the mucous membrane of the caecum was very pale but otherwise quite normal in appearance. On the other pale but otherwise quite normal in appearance. hand, the congested con foreign body in the intestine. The above. There was no foreign body in the intestine. The large intestine was very contracted and pale, and seemed mon to the whole of the large bowel. The appendix was healthy. It was situated in the right iliac fossa, where the caecum was also found in its usual position. There was no evidence of any congenital maldevelopment. Meckel's divertievidence of any conger There was no sign of internal hernia in culum was not found. There was no sign of mesenteric vessels any part of the abdomen. The superior mesenteric vessels were normal in appearance, and there was no sign of portal thrombosis or embolism. The pancreas was healthy, and there was no sign of fat necrosider was contracted and the urine con tained no sugar. The liver and gall bladder and its ducts were healthy, and there were no adhesions around thess parts. Tre healominal aorta and inferior vena cava presented no sign of disease. The spleen was rather large but otherwise was normal. There was no fluid in the abdominal cavity.

From the history of the case and the post-mortem appearances, it is evident that it was one of acute volvulus of the ileum. The constriction was definite below at the ileo-caecal valve; above it was difficult to say where the gut was gripped, as no sign of constriction could be found 
In doing a post.mortem examination of this kind, one naturally looks first for the commoner causes of acute disease, and the appendix is early searched for on this account. Unfortunately, the moving of the intestines in the search for the appendix disturbed the position of the ileum, and the position of the upper limit of the volvulus must remain more or less hypothetical. The extreme distension affected about $20 \mathrm{in}$. of the ileum, and the upper limit of the volvulus was probably in this position. In recorded cases of volvulus of the ileum, both the upper and lower limits seem in some cases to have been more or less indefinite.

Death supervened in five hours in this case, and was undoubtedly due to shock following an acute strangulation of a comparatively large amount of bowel, and that bowel part of the small intestine which is of much more vital importance than the large. From the immense size this part of intestine reached, and the amount of congested blood in it, there was practically a severe haemorrhage into it, as far as the general circulation was concerned. This must have greatly increased the already severe shock from which the patient was suffering.

Shock is defined as a condition of exhaustion of the vasomotor centre. Crile has shown that if the abdomen of an animal is opened and the intestines exposed, a fall of blood pressure occurs. This is associated with a vascular dilatation of the splanchnic area, and a marked degree of shock occurs. The animal bleeds into its own portal system. Anaemia of the vasomotor centre results, and sets up a general vasomotor paralysis, which increases as the vasomotor centre becomes exhausted. Treves states that " the grave initial symptoms of intestinal obstruction do not depend upon the fact that the bowel is obstructed, but upon the severe injury to the sympathetic nerves involved." Crile's observations show that this injury acts by producing an inhibition of the vaso-constrictor nerves, and ultimate paralysis of these nerves by exhaustion of the vasomotor centre. The resulting dilatation of the vessels of the splanchnic area causes a great fall in the systemic blood pressure and coincident shock.

In the case of this man there was an added haemorrhage into the part of the intestine forming the volvulus, thus increasing the factors causing shock, and making it much more profound owing to the added anaemia of the brain.

I can find no record of death ensuing in so short a time from intestinal obstruction, although several cases have been recorded where death ensued within twenty-four hours. Dr. Bryant suggests that the man was one who did not complain much, and that he may have had some pain earlier than the time stated.

The part of the ileum involved in this case, the lower part, is most frequently the seat of volvulus when it occurs in the small intestine. Treves says "the majority of patients exhibiting this form of volvulus have been males with a mean age of 25 years." Cases of volvulus of the small intestine in cases of typhoid fever, simulating perforation, are mentioned by Roberts; the symptoms were severe abdominal pain and collapse. Eliot has recorded a case in which a volvulus of the ileum, associated with one of the pelvic colon, caused great shock, with a pulse of 140 , thready and very weak, coldness and lividity, and apathy, but the facial expression showed intense suffering. In the case I have recorded the symptoms of shock were present to such a degree as to cause death within a few hours.

\section{TWO CASES OF APPENDICITIS.}

\section{By H. GOODWYN, F.R.C.S.EDIN.,} BOVEY TRACEY.

The following two cases of appendicitis presenting some unusual features are, I think, worth recording.

$$
\text { CASE I. }
$$

Mrs. C., aged 36, had for a period of two months repeated attacks of severe appendicular colic, accompanied on each occasion by a rise of temperature- $101^{\circ}$ to $102^{\circ}$-with severe abdominal pain, referred at first to the umbilical region, and latterly localized to the region of the appendix, the maximum point of tenderness being over McBurney's point. These attacks subsided under hot fomentations, rest in bed, etc., but the attacks became more frequent and intervals shorter; also they were more severe in character. The patient was three months pregnant, but in the light of the increasing severity of the pain, and frequency of attacks, it was decided to operate, and at the worst the risk of miscarriage was less than the possibility of the formation of an abscess.

Operation.-The caecum was much bound down by adhesions, and the tip of the appendix was adherent to the pelvic brim. There was great difficulty in withdrawing the caecum sufficiently to expose the base of the appendix, and to free the appendix itself, owing to numerous adhesions, but eventually this was accomplished, and a long inflamed appendix was removed, and the stump invaginated by purse-string suture, and covered by Lembert's sutures.

The subsequent history was uneventful; on the next day temperature and pulse were normal, and on the fourteenth day the patient returned home; no signs of uterine disturbance had occurred, and she remained well after six weeks. On opening the appendix, a nematode worm about $1 \frac{1}{2}$ in. in length was found, and there was a haemorrhagic condition of the mucous. lining of the whole organ, and an ulcer commencing to form on the floor, which would in all probability have gone on very shortly to perforation. I think the result amply justified the possible risk of cutting short the pregnancy.

\section{CASE II.}

A child aged 4, who for about twelve months had had attacks nome very severe, of abdominal pain without localized tenderness. principally referred to the umbilical region. Six weeks before operation she had a severe attack of pain, with rise of temperature to $102^{\circ}$; vomiting and marked constipation. There was no tenderness over the appendix at first; in about four days the pain was localized. The appendix, which was much inflamed, $4 \frac{1}{3}$ in. long, and considerably swollen, was isolated without difficulty. It was treated by invagination with purse-string suture, and closed over with Lembert's sutures. The recovery was uneventful; there was no rise of temperature or pulse, and the stitches were removed on the eighth day.

On opening the appendix a haemorrhagic condition of the mucous lining was seen, and a small fishbone, about ${ }_{5}^{5}$ in. long. lying free in the lumen. There was also evidence of an old kink, as represented by a transverse band of scar tissue.

Both these cases are illustrative of the advantage of early operation when symptoms of colic recur without. obvious cause; in the second case, though the temperature: and pulse became normal in a few days, during the whole five weeks there was never complete freedom from slight. attacks of pain, sometimes lasting only a few minutes, norfrom tenderness on deep pressure over the appendix.

The technique I practised was by incision through the skin over the outer border of the rectus, which was pushed inwards without splitting its fibres. 1 think this method" has distinct advantages over the gridiron method, as especially in the first case it would have been nearly impossible to obtain sufficient room for the necessary manipulations without dividing the fibres of the internab oblique and transversalis. I followed Professor von Eiselsberg, of Vienna, in painting the skin with a spirit. lotion of iodine liniment both before the incision and after the stitches were introduced.

\section{A CASE OF VERY LARGE GALL BLADDER. SLCCESSFULLY TREATED BY EXCISION.*}

By F. W. COLLINSON, M.D.Edin., M.R.C.P.LOND.\% F.R.C.S.EDIN.

HONORARY MEDICAL:OFFICER, PRESTON ROYAL INFIRMARY.

THIs case is remariable owing to the immense size of the gall bladder, being amongst the largest on record.

Mrs. S., aged 31, was sent to me by Dr. Callanan of Longridge on October 14th, 1908, suffering from general abdominas distension and pain in the right side of the abdomen.

\section{History.}

There was nothing of note in the family history, as all were healthy; the mother had died in childbirth. The patient herself had always been in good health, though pale. She had two. children, now living and healthy, born six and three years ago, with one miscarriage in between them.

The history of the present affection appeared to date from August, 1907, when she had a fall, for soon after she began to vomit each day, and in December she remained in bed on that account. She distinctly said there was then no swelling of the abdomen. In January she observed a swelling of the lower part. of the abdomen towards the right side. On February 13th she: had greatly increased in size, and was somewhat emaciated and markedly jaundiced. She was tapped, and 25 pints of fluid was drawn off, and the fluid must have been similar to that removed afterwards at the operation. Dr. Callanan was able to "feel the liver for 2 in. or 3 in. below the ribs," but he also wrote, rather prophetically, "this may, of course, be the sac of

Notes read, and patient shown, to the Clinical Branch of the Preston Medico-Ethical Society, February 3rd, 1909 